\title{
Invariant-based inverse engineering of time-dependent, coupled harmonic oscillators
}

\author{
A. Tobalina, ${ }^{1, *}$ E. Torrontegui $\odot,{ }^{2,3}$ I. Lizuain, ${ }^{4}$ M. Palmero $\odot,{ }^{5,6}$ and J. G. Muga ${ }^{1}$ \\ ${ }^{1}$ Department of Physical Chemistry, University of the Basque Country UPV/EHU, Apdo 644, Bilbao, Spain \\ ${ }^{2}$ Departamento de Física, Universidad Carlos III de Madrid, Avda. de la Universidad 30, 28911 Leganés (Madrid), Spain \\ ${ }^{3}$ Instituto de Física Fundamental IFF-CSIC, Calle Serrano 113b, 28006 Madrid, Spain \\ ${ }^{4}$ Department of Applied Mathematics, University of the Basque Country UPV/EHU, 20018 Donostia-San Sebastian, Spain \\ ${ }^{5}$ Department of Applied Physics I, University of the Basque Country, UPV/EHU, 48013 Bilbao, Spain \\ ${ }^{6}$ Science and Math Cluster, Singapore University of Technology and Design, 8 Somapah Road, 487372 Singapore
}

(Received 27 July 2020; accepted 28 October 2020; published 14 December 2020)

\begin{abstract}
Two-dimensional (2D) systems with time-dependent controls admit a quadratic Hamiltonian modeling near potential minima. Independent, dynamical normal modes facilitate inverse Hamiltonian engineering to control the system dynamics, but some systems are not separable into independent modes by a point transformation. For these "coupled systems" 2D invariants may still guide the Hamiltonian design. The theory to perform the inversion and two application examples are provided: (i) We control the deflection of wave packets in transversally harmonic wave guides and (ii) we design the state transfer from one coupled oscillator to another.
\end{abstract}

DOI: 10.1103/PhysRevA.102.063112

\section{INTRODUCTION}

Controlling the motional dynamics of quantum systems is of paramount importance for fundamental science and quantum-based technologies [1]. In particular, controlling the evolution of interacting quantum systems is crucial to design logic gates, one of the key elements of a quantum computer [2]. Often the external driving needs to be fast, but also gentle, to avoid excitations. Slow adiabatic driving is gentle in this sense, but it exposes the system for long times to control noise, heating, and perturbations. Shortcuts to adiabaticity (STA) are techniques to reach, via fast nonadiabatic routes, the results of slow adiabatic processes $[3,4]$.

A distinction can be made between STA methods that keep the structure of some Hamiltonian form and design the time dependence of the controls, e.g., using invariants [5] and those techniques that add new terms, e.g., counterdiabatic driving [6]. Both may be useful depending on system-dependent practical considerations. A frequent problem with added terms is the difficulty to implement them, whereas a limitation of structure-preserving, invariant-based methods is that they need Hamiltonian-invariant pairs with specific forms, such as the Lewis-Leach family of Hamiltonian-invariant pairs [7], to go beyond brute-force parameter optimization $[3,4]$.

Here we shall deal with two-dimensional (2D) systems with quadratic Hamiltonians, found in particular in smalloscillation regimes of ultracold atom physics. In fact quadratic Hamiltonians are ubiquitous as they represent the systems

\footnotetext{
*ander.tobalina@ehu.eus

Published by the American Physical Society under the terms of the Creative Commons Attribution 4.0 International license. Further distribution of this work must maintain attribution to the author(s) and the published article's title, journal citation, and DOI.
}

near potential minima [8]. So far, invariant based STA have only been developed for 2D systems with independent "dynamical normal modes" [9]. When the two dynamicalmode motions separate, inverse engineering the dynamics to perform some fast operation free from final excitations is relatively easy: each of the time-dependent effective oscillators implies a one-dimensional Hamiltonian-invariant "Lewis-Leach" pair [7] for which inverse engineering can be performed. The two oscillators have to be driven simultaneously with common controls but, among the plethora of parameter trajectories, it is possible to find the ones that satisfy simultaneously the boundary conditions imposed on both oscillators. This strategy has been successfully applied to design the driving of different operations on two trapped ions such as transport or expansions $[9,10]$, separation of two equal ions in double wells [11], phase gates [12], or dynamical exchange cooling [13].

This decomposition though, may not always be possible. Lizuain et al. [14] described the condition for which a point transformation of coordinates decouples the instantaneous modes leading to truly independent dynamical normal modes for two time-dependent harmonic oscillators: the principal axes of the potential should not rotate in the 2D space.

This work extends the domain of systems and processes that can be controlled by invariant-based inverse engineering to those problems where the effective $2 \mathrm{D}$ potential rotates and the normal mode motions remain coupled. Solutions to the ensuing control problem exist that depend on the system and/or the operation, such as taking refuge in a perturbative regime [12], adding terms to cancel the inertial effects [14], increasing the number of time-dependent controls to uncouple the modes [13], or using more complex, nonpoint transformations to find independent modes [15]. Here we explore instead the use of 2D dynamical invariants associated with the coupled Hamiltonian. 
The paper is organized as follows: First we introduce the model and its dynamical normal modes in Sec. II. Then we present the invariant we will use to inverse engineer the control fields in Sec. III. The first application, in Sec. IV, is the control of longitudinal energy in 2D deflected wave guides. Section V describes further control possibilities for 2D waveguides, and Sec. VI deals with a second type of application: controlled state transfer between oscillators. The paper ends with a discussion in Sec. VII.

\section{HAMILTONIAN MODEL}

\section{Consider the Hamiltonian}

$$
H(t)=\frac{p_{1}^{2}}{2}+\frac{p_{2}^{2}}{2}+\frac{1}{2} \omega_{1}^{2}(t) q_{1}^{2}+\frac{1}{2} \omega_{2}^{2}(t) q_{2}^{2}-\gamma(t) q_{1} q_{2} .
$$

We use throughout dimensionless variables such that no mass factors or $\hbar$ appear explicitly. Equation (1) describes different physical systems, such as a single particle in a 2D potential, or two coupled harmonic oscillators on a line. Other systems different from (one or two) particles but driven by Hamiltonians of the form (1) are, e.g., coupled superconducting qubits [16-20] or optomechanical oscillators [21-23]. All these systems are analogous to each other but, arguably, the single particle in a $2 \mathrm{D}$ potential is easiest to visualize so we shall use a terminology (such as longitudinal and transversal directions for principal axes, rotations...) borrowed from that system. Indeed, our first example, see below, deals with a single particle.

The Hamiltonian (1) may be instantaneously diagonalized by "rotated" variables [14]

$$
\left(\begin{array}{l}
q_{l} \\
q_{t}
\end{array}\right)=A(t)\left(\begin{array}{l}
q_{1} \\
q_{2}
\end{array}\right), \quad\left(\begin{array}{l}
p_{l} \\
p_{t}
\end{array}\right)=A(t)\left(\begin{array}{l}
p_{1} \\
p_{2}
\end{array}\right),
$$

$$
\begin{aligned}
\text { where } A(t)= & \left(\begin{array}{cc}
\cos \theta(t) & \sin \theta(t) \\
-\sin \theta(t) & \cos \theta(t)
\end{array}\right) \text {, and } \\
& \theta(t)=\frac{1}{2} \arctan \left(\frac{2 \gamma(t)}{\omega_{2}^{2}(t)-\omega_{1}^{2}(t)}\right) .
\end{aligned}
$$

Subscripts $l$ and $t$ stand for "longitudinal" and "transversal". The original Hamiltonian, expressed in terms of the new variables, is

$$
\begin{gathered}
H=\frac{p_{l}^{2}}{2}+\frac{p_{t}^{2}}{2}+\frac{1}{2} \Omega_{l}^{2} q_{l}^{2}+\frac{1}{2} \Omega_{t}^{2} q_{t}^{2}, \\
\Omega_{l}^{2}=\left(\omega_{1}^{2}+\omega_{2}^{2}-\Lambda\right) / 2, \quad \Omega_{t}^{2}=\left(\omega_{1}^{2}+\omega_{2}^{2}+\Lambda\right) / 2,
\end{gathered}
$$

where $\Lambda(t)=\sqrt{4 \gamma^{2}(t)+\left[\omega_{2}^{2}(t)-\omega_{1}^{2}(t)\right]^{2}}$.

The formal decoupling in Eq. (4) is a mirage. $H$ is not the Hamiltonian that describes the dynamics in the rotated variables $\left\{p_{l}, p_{t}, q_{l}, q_{t}\right\}[14,24]$. In general the dependence of $A(t)$ on time couples dynamically the "instantaneous normal modes", i.e., the normal modes that would separate the motion if the Hamiltonian kept for all times the values that the parameters have at a particular instant. In the moving frame the oscillators are coupled by a term proportional to $\dot{\theta}=d \theta / d t$ [14]. Some peculiar, but physically significant relations between $\omega_{1}(t), \omega_{2}(t)$, and $\gamma(t)$ can make $\theta(t)$ time independent. Here we consider instead the scenario where $\theta(t)$ changes with time. This is unavoidable if the process we want to implement implies boundary conditions for the parameters such that $\theta(0) \neq \theta\left(t_{f}\right)$, as in the examples below.

\section{2D INVARIANT}

Urzúa et al. [8], generalizing previous results in 1D [25,26] and the work in Ref. [27] for classical coupled oscillators, see also Ref. [28], have recently found that the linear combination of operators (dots stand for time derivatives hereafter)

$$
G(t)=u_{1}(t) p_{1}-\dot{u}_{1}(t) q_{1}+u_{2}(t) p_{2}-\dot{u}_{2}(t) q_{2},
$$

satisfies the invariant equation $i \partial G / \partial t-[H, G]=0$, provided $u_{1}$ and $u_{2}$ satisfy

$$
\ddot{u}_{1}+\omega_{1}^{2}(t) u_{1}=\gamma(t) u_{2}, \quad \ddot{u}_{2}+\omega_{2}^{2}(t) u_{2}=\gamma(t) u_{1},
$$

which are classical equations of motion driven by a Hamiltonian (1). For any state driven by $H(t),\langle G(t)\rangle$ is the sum of two Wronskians $W_{1}\left[u_{1}(t),\left\langle q_{1}\right\rangle(t)\right]+W_{2}\left[u_{2}(t),\left\langle q_{2}\right\rangle(t)\right]$, where all functions in their arguments evolve as Eq. (7). The geometrical meaning of $W_{i}(t)$ is an "oriented" phase-space area formed by phase-space points $U_{i}(t)=\left\{u_{i}(0), \dot{u}_{i}(t)\right\}, Q_{i}(t)=$ $\left\{\left\langle q_{i}\right\rangle(t),\left\langle p_{i}\right\rangle(t)\right\}$ and the origin $O_{i}=\left\{0_{i}, 0_{i}\right\}$. We consider two phase spaces, $i=1,2$, one for each oscillator. $W_{i}(t)$ is plus or minus the area $A_{i}(t)$ of the triangle formed by $U_{i}, Q_{i}$ and $O_{i}$ for each phase space, depending on whether going from $U_{i}$ to $Q_{i}$ needs an anticlockwise or clockwise displacement. For $\gamma=0$, the two areas (and Wronskians) remain constant in time. When $\gamma \neq 0$ the individual Wronskians are not conserved. The conserved quantities are now $W_{i}(t)-\int_{0}^{t} \dot{W}_{i}\left(t^{\prime}\right) d t^{\prime}=W_{i}(0)$, i.e., the initial phase-space oriented areas. The added terms cancel each other, namely, $\dot{W}_{1}=$ $-\dot{W}_{2}=\left(u_{1}\left\langle q_{2}\right\rangle-\left\langle q_{1}\right\rangle u_{2}\right) \gamma$, so that the sum $W_{1}(t)+W_{2}(t)$ is the sum of oriented areas and it is constant. This result is a particular case of the preservation of sums of oriented areas in classical Hamiltonian systems [29].

We construct from $G$ a quadratic invariant that may become proportional to some relevant energy at boundary times by choosing specific boundary conditions for the $u_{i}$ and $\dot{u}_{i}$, $I=\frac{1}{2} G^{\dagger} G$. Designing the $u_{i}$ we may manipulate the invariants and therefore the dynamics. From the $u_{i}$ we can as well get the Hamiltonian as demonstrated in the following application examples.

\section{CONTROLLED DEFLECTION}

A single particle is launched along a potential "wave guide", which is harmonic in the transversal direction. Our goal is to deflect it, that is, manipulate the potential to change the waveguide direction, controlling the input/output scaling factor of the longitudinal velocity. To have waveguide potentials at the boundary times $t_{b}=0, t_{f}$ we impose

$$
\gamma\left(t_{b}\right)=\omega_{1}\left(t_{b}\right) \omega_{2}\left(t_{b}\right) .
$$

As a consequence, $\Omega_{l}\left(t_{b}\right)=0$ and $\Omega_{t}\left(t_{b}\right)=\left[\omega_{1}^{2}\left(t_{b}\right)+\right.$ $\left.\omega_{2}^{2}\left(t_{b}\right)\right]^{1 / 2}$. Thus, at boundary times, the potential is a harmonic "waveguide" with longitudinal direction defined by the angle $\theta\left(t_{b}\right)=\arctan \left[\omega_{1}\left(t_{b}\right) / \omega_{2}\left(t_{b}\right)\right]$. The deflection angle $\Delta \theta=\theta\left(t_{f}\right)-\theta(0)$ can take any value between 0 and $\pi / 2$ for $\theta\left(t_{f}\right) \geqslant \theta(0)$. The condition (8) in Eq. (7) implies that 
TABLE I. Initial and final frequencies and angles defining the wave guides for $\gamma$-constant and $\omega_{2}$-constant protocols. The deflection angle $\Delta \theta=\theta\left(t_{f}\right)-\theta(0)$ determines the ratio $\omega_{2}(0) / \omega_{1}(0)$.

\begin{tabular}{lll}
\hline \hline & Initial wave guide & \multicolumn{1}{c}{ Final wave guide } \\
\hline$\gamma$ const. & $\omega_{1}(0)$ & $\omega_{1}\left(t_{f}\right)=\omega_{2}(0)$ \\
& $\omega_{2}(0)$ & $\omega_{2}\left(t_{f}\right)=\omega_{1}(0)$ \\
& $\Omega_{t}(0)$ & $\Omega_{t}\left(t_{f}\right)=\Omega_{t}(0)$ \\
$\omega_{2}$ const. & $\omega_{1}(0)$ & $\omega_{1}\left(t_{f}\right)=\omega_{2}^{2}(0) / \omega_{1}(0)$ \\
& $\omega_{2}(0)$ & $\omega_{2}\left(t_{f}\right)=\omega_{2}(0)$ \\
& $\Omega_{t}(0)$ & $\Omega_{t}\left(t_{f}\right)=\frac{\omega_{2}(0)}{\omega_{1}(0)} \Omega_{t}(0)$ \\
\hline \hline
\end{tabular}

$\ddot{u}_{1,2}\left(t_{b}\right)=0$, which also gives

$$
u_{1}\left(t_{b}\right) \omega_{1}\left(t_{b}\right)=u_{2}\left(t_{b}\right) \omega_{2}\left(t_{b}\right),
$$

i.e., the reference trajectories must start and end at $q_{t}\left(t_{b}\right)=0$, on the axis of the waveguide. If the frequencies at $t_{b}$ are fixed, either $q_{l}\left(t_{b}\right)$, or one of the $u_{i}\left(t_{b}\right)$ can still be chosen freely.

Rewriting the invariant $G$ in terms of the rotated variables $\left\{q_{t}, q_{l}\right\}$ and imposing $\dot{u}_{1,2}\left(t_{b}\right)=0$ we find that

$$
G\left(t_{b}\right)=\frac{u_{2}\left(t_{b}\right)}{\sin \theta\left(t_{b}\right)} p_{l}, \quad I\left(t_{b}\right)=\left[\frac{u_{2}\left(t_{b}\right)}{\sin \theta\left(t_{b}\right)}\right]^{2} \frac{p_{l}^{2}}{2},
$$

i.e., $I\left(t_{b}\right)$ is proportional to the longitudinal energy.

With Eq. (10) we get

$$
\left\langle p_{l}\left(t_{f}\right)\right\rangle=F\left\langle p_{l}(0)\right\rangle, \quad E_{l}\left(t_{f}\right)=F^{2} E_{l}(0),
$$

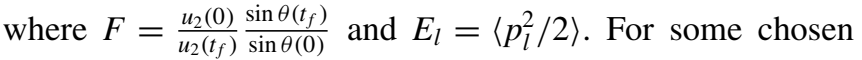
deflection angle $\Delta \theta$ and waveguide frequencies $\Omega_{t}\left(t_{b}\right)$ we may impose any scaling factor $F$ by manipulating the ratio $u_{2}(0) / u_{2}\left(t_{f}\right)$, which allows us to set any desired velocity scaling, that is, any ratio between the incoming and the outgoing average velocity of the wave packet. This scaling factor will affect all wave packets, and the deflection angle $\Delta \theta$ and the waveguide compression/expansion factors [ratio between $\Omega_{t}(0)$ and $\left.\Omega_{t}\left(t_{f}\right)\right]$ can be chosen independently.

The Hamiltonian parameters are found inversely from Eq. (7). We choose $u_{1,2}=\sum_{k=0}^{5} \alpha_{k}^{(1,2)}\left(t / t_{f}\right)^{k}$, with coefficients fixed so that $\dot{u}_{1,2}\left(t_{b}\right)=\ddot{u}_{1,2}\left(t_{b}\right)=0$, and the $u_{1,2}\left(t_{b}\right)$ are consistent with Eq. (9).

There are three external parameters, $\omega_{1}(t), \omega_{2}(t)$, and $\gamma(t)$, but two coupled equations in Eq. (7). Thus we may fix one of the external parameters or some combination. We consider two simple, not exhaustive, possibilities: (i) $\gamma$ constant, so initial and final $\Omega_{t}$ coincide; and (ii) $\omega_{2}$ constant, which implies a compression (transverse focusing useful to avoid transversal excitation) of the final wave guide with respect to the initial one, see Table I.

The initial state chosen for the numerical examples is a product of the ground state of the transversal harmonic oscillator and a minimum-uncertainty-product Gaussian in the longitudinal direction centered at $q_{l 0}$, with initial momentum $p_{l 0}, \psi_{l}\left(q_{l}, t=0\right)=[\sigma \sqrt{2 \pi}]^{-1 / 2} e^{i p_{l 0} q_{l}} e^{-\left(q_{l}-q_{l}\right)^{2} /\left(4 \sigma^{2}\right)}$. Firstly, we design a process that interchanges $\omega_{1}(t)$ and $\omega_{2}(t)$ with $\Delta \theta=\pi / 4$ and constant $\gamma$, conceived to preserve the initial longitudinal velocities in the outgoing waveguide,
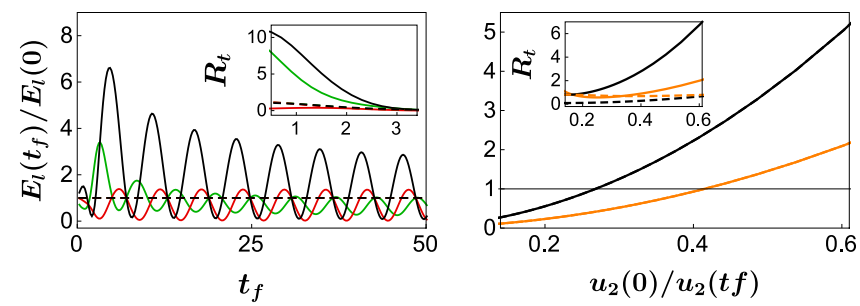

FIG. 1. Ratio of final to initial longitudinal energy for different process times $t_{f}$ (a) and for different scaling factors $u_{2}(0) / u_{2}\left(t_{f}\right)(\mathrm{b})$. The insets show the scaled transversal excitation $R_{t}=\Delta E_{t} / \Omega_{t}\left(t_{f}\right)$. (a) initial longitudinal Gaussian wave packet with $2^{1 / 2} \sigma=1, p_{l 0}=$ 1 , and $q_{l 0}=-4$ (green), $q_{l 0}=0$ (red), and $q_{l 0}=4$ (black). $\Delta \theta=$ $\pi / 4$ starting from $\omega_{1}(0)=1$ and $\omega_{2}(0)=2.41$, using linear ramps (solid lines) and an invariant-based protocol for $\gamma$ constant that produces $E_{l}\left(t_{f}\right)=E_{l}(0)$ (dashed lines). (b) Initial longitudinal Gaussian wave packet centered at the origin with $p_{l 0}=1$ and $2^{1 / 2} \sigma=1$. $\Delta \theta=\pi / 4$ with $\omega_{1}(0)=1$ and $\omega_{2}(0)=2.41$ (orange curves), and $\Delta \theta=\pi / 3$ with $\omega_{1}(0)=1$ and $\omega_{2}(0)=3.73$ (black curves) for constant $-\gamma$ processes (solid lines) and constant- $\omega_{2}$ processes (dashed lines, overlapping with solid lines in main figure). See Table I for values at $t=t_{f}$.

$E_{l}\left(t_{f}\right)=E_{l}(0)$, and use linear ramps (a control field that evolves linearly between the boundary values) for the same boundary waveguides as a benchmark to compare the performance of the invariant-based protocol.

Figure 1(a) depicts the final longitudinal energy. For the linear ramps it oscillates with operation time. The envelope for the minima is at zero but the maximum tends for long times to some value that depends on the initial wave packet. Contrast this with the full stability of the invariant-lead processes. They guarantee a fixed result, the final longitudinal energy being identical to the initial one for any initial wave packet. The transversal excitation by the linear ramps in fast processes increases considerably as the initial wave packet deviates from the origin, while the transversal excitation in the invariantbased protocol is, in general, small and much more stable.

Figure 1(b) verifies that, for some chosen deflection angle, we can scale the final longitudinal energy at will in both scenarios ( $\gamma$ or $\omega_{2}$ constant). Since the invariant does not control the transversal direction, the transversal energy may be excited, but it still depends on the design of the $u_{i}(t)$, see the inset of Fig. 1(b). Such dependence may be exploited to minimize the transversal excitation and even suppress it in some cases (notice that the $\pi / 3$ rotation with constant $\omega_{2}$ produces zero transversal excitation for a given relation between the boundary values of $u_{2}$ ). Figure 2 provides snapshots of

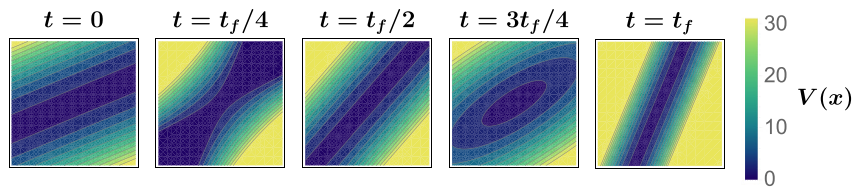

FIG. 2. Snapshots of the top view of the $2 \mathrm{D}$ potential for $E_{l}\left(t_{f}\right)=$ $E_{l}(0) / 2$ with constant $\omega_{2} . \omega_{1}(0)=1$ and $\omega_{2}=2.41$, deflection angle $\Delta \theta=\pi / 4\left(\omega_{1}\left(t_{f}\right)=2.41^{2}\right)$ and process time $t_{f}=1$. The transversal frequency is compressed 2.41 times, from $\Omega_{t}(0)=2.61$ to $\Omega_{t}\left(t_{f}\right)=$ 6.29 , see Table I. 
the evolution of the $2 \mathrm{D}$ potential for a $\omega_{2}$-constant processes that slows down the particle by a factor of 2 with deflection $\Delta \theta=\pi / 4$.

\section{COMMUTATION OF $H\left(t_{b}\right)$ AND $I\left(t_{b}\right)$ AND OTHER BOUNDARY CONDITIONS}

Note that the necessary condition for the Hamiltonian and the invariant to commute at boundary times is precisely the waveguide condition in Eq. (8) (together with the auxilary equations in Eq. (7)). In the case of a potential waveguide, however, the eigenvectors of $I\left(t_{b}\right)$ are highly degenerate, since a longitudinal plane wave multiplied by an arbitrary function of $q_{t}$ is a valid eigenvector with the same eigenvalue. This means that even if $I\left(t_{b}\right)$ commutes with $H\left(t_{b}\right)$ and shares some eigenvectors with $H\left(t_{b}\right)$ the vast majority of them are not eigenvectors of $H\left(t_{b}\right)$. This phenomenon, i.e., the existence of eigenvectors of one operator not shared with the other one, is well known and is explained in detail in Ref. [30].

Thus, commutativity of $H\left(t_{b}\right)$ and $I\left(t_{b}\right)$ plays a lesser role in the 2D scenario, compared to the use of invariants for inverse engineering in 1D [4], and may in fact be abandoned for different applications. For example, note the following alternative sets of boundary conditions and corresponding quadratic invariants:

$$
\begin{aligned}
\dot{u}_{i}\left(t_{b}\right) & =0, u_{1}\left(t_{b}\right) \omega_{2}\left(t_{b}\right)=-u_{2}\left(t_{b}\right) \omega_{1}\left(t_{b}\right), \\
I\left(t_{b}\right) & =\frac{u_{2}^{2}\left(t_{b}\right)}{\cos ^{2} \theta\left(t_{b}\right)} \frac{p_{t}^{2}}{2},
\end{aligned}
$$

where $i=1,2$ and the invariant at the boundary time $t_{b}$ is proportional to the transversal kinetic energy. With these boundary conditions we could control and scale the transverse kinetic energy. As well,

$$
\begin{aligned}
u_{i}\left(t_{b}\right) & =0, \dot{u}_{1}\left(t_{b}\right) \omega_{1}\left(t_{b}\right)=\dot{u}_{2}\left(t_{b}\right) \omega_{2}\left(t_{b}\right), \\
I\left(t_{b}\right) & =\frac{\dot{u}_{2}^{2}\left(t_{b}\right)}{\sin ^{2} \theta\left(t_{b}\right)} \frac{q_{l}^{2}}{2},
\end{aligned}
$$

which allows us to scale the longitudinal coordinate, e.g., to focus or defocus, or

$$
\begin{aligned}
u_{i}\left(t_{b}\right) & =0, \dot{u}_{1}\left(t_{b}\right) \omega_{2}\left(t_{b}\right)=-\dot{u}_{2}\left(t_{b}\right) \omega_{1}\left(t_{b}\right), \\
I\left(t_{b}\right) & =\frac{\dot{u}_{2}^{2}\left(t_{b}\right)}{\cos ^{2} \theta\left(t_{b}\right)} \frac{q_{t}^{2}}{2},
\end{aligned}
$$

where the invariant at the boundary is proportional to the transverse potential energy.

Even more generally, the boundary conditions imposed on the $u_{i}(t)$ and their derivatives do not need to be of the same type at $t=0$ and $t_{f}$, i.e., for longitudinal momenta or positions at both boundary times, of for transverse momenta or positions at both boundary times. Designing $u_{i}(t)$ so as to satisfy at $t=0$ and $t_{f}$ different boundary condition types opens several control possibilities such as, for example, driving the initial longitudinal energy into final transversal kinetic energy or vice versa.

\section{STATE TRANSFER}

Up to now we have considered real $u_{j}(t)$, but the coupled Newton's equations admit purely real and purely imaginary solutions combined into complex solutions. Exploiting this complex structure, $u_{i}=u_{i}^{R}+i u_{i}^{I}$, leads to interesting forms of the invariant. In particular the invariant may become proportional to the uncoupled Hamiltonians at boundary times, enabling energy transfer form one oscillator to the other. Processes that exchange the state, or some property of it, between coupled systems are highly relevant for the development of quantum technologies. They have been extensively studied in the context of quantum computation and communication [31,32] and also addressed in experiments [33,34]. State transfer between coupled harmonic oscillators has also been thoroughly explored [35-37]. Here we develop a protocol that induces energy exchange between oscillators by inverse engineering the control fields that govern the system.

Let us first drop the waveguide condition (8) and go back to the laboratory frame variables $\left\{q_{1}, q_{2}\right\}$. Defining annihilation operators in the usual manner, $a_{i}(t)=\sqrt{\omega_{i}(t) / 2} q_{i}+$ $i p_{i} / \sqrt{2 \omega_{i}(t)}, \quad i=1,2, G$ in Eq. (6) may become $a_{1}$ or $a_{2}$ by certain choices of the $u_{j}$. Let us choose at initial time

$$
u_{1}(0)=i c_{0} / \sqrt{2 \omega_{1}(0)}, \quad \dot{u}_{1}(0)=-c_{0} \sqrt{\omega_{1}(0) / 2},
$$

and $u_{2}(0)=\dot{u}_{2}(0)=0$ with $c_{0}$ real. This implies $G(0)=$ $c_{0} a_{1}(0)$, and $I(0)=c_{0}^{2} a_{1}^{\dagger}(0) a_{1}(0) / 2$. Instead, at final time we impose

$$
u_{2}\left(t_{f}\right)=i c_{0} / \sqrt{2 \omega_{2}\left(t_{f}\right)}, \quad \dot{u}_{2}\left(t_{f}\right)=-c_{0} \sqrt{\omega_{2}\left(t_{f}\right) / 2},
$$

together with $u_{1}\left(t_{f}\right)=\dot{u}_{1}\left(t_{f}\right)=0$, so that $G\left(t_{f}\right)=c_{0} a_{2}\left(t_{f}\right)$, and $I\left(t_{f}\right)=c_{0}^{2} a_{2}^{\dagger}\left(t_{f}\right) a_{2}\left(t_{f}\right) / 2$. The same constant $c_{0}$ appears in Eqs. (15) and (16) because the solutions of Eq. (7) must satisfy $\frac{d}{d t}\left\{\operatorname{Im}\left[u_{1}^{*}(t) \dot{u}_{1}(t)+u_{2}^{*}(t) \dot{u}_{2}(t)\right]\right\}=0$ [27].

The choice $c_{0}^{2} / 2=\omega_{1}(0)$, together with the mentioned boundary conditions give $I(0)=H_{1}(0)$ and $I\left(t_{f}\right)=$ $\left[\omega_{1}(0) / \omega_{2}\left(t_{f}\right)\right] H_{2}\left(t_{f}\right)$, where we define the "uncoupled Hamiltonians" $H_{j}(t) \equiv \omega_{j}(t) a_{j}^{\dagger}(t) a_{j}(t)$. Eigenstates of $H_{1}(0)$ may thus be mapped into eigenstates of $H_{2}\left(t_{f}\right)$ by proper inverse engineering of the $u_{j}(t)$. If $\omega_{1}(0)=\omega_{2}\left(t_{f}\right)$,

$$
\left\langle H_{1}(0)\right\rangle=\langle I(0)\rangle=\left\langle I\left(t_{f}\right)\right\rangle=\left\langle H_{2}\left(t_{f}\right)\right\rangle
$$

for all initial wave packets. (Any other scale factor may be chosen.) The system (7), which now involves four real functions, $u_{1}^{R}(t), u_{1}^{I}(t), u_{2}^{R}(t), u_{2}^{I}(t)$, has to be solved inversely for $\omega_{1}(t), \omega_{2}(t)$ and $\gamma(t)$. The inversion is done following techniques developed for trapped ions [12] or systems [38]. Assuming that the values of the control parameters at boundary times are set, we start by designing a $\gamma(t)$ that satisfies the boundary values $\gamma\left(t_{b}\right)$ and that has zero first and second derivatives at the boundaries for smoothness. We use a sumof-cosines ansatz

$$
\gamma(t)=\sum_{k=0}^{4} a_{k} \cos \left(\frac{k \pi t}{t_{f}}\right),
$$

which meets the boundary conditions with just five terms. The coefficient $a_{4}$ is left free for now. Then we design the imaginary part of the dynamics, again using sums of cosines

$$
\begin{aligned}
& u_{1}^{I}(t)=\sum_{i=0}^{6} b_{i} \cos \left(\frac{i \pi t}{t_{f}}\right), \\
& u_{2}^{I}(t)=\sum_{j=0}^{6} c_{j} \cos \left(\frac{j \pi t}{t_{f}}\right) .
\end{aligned}
$$



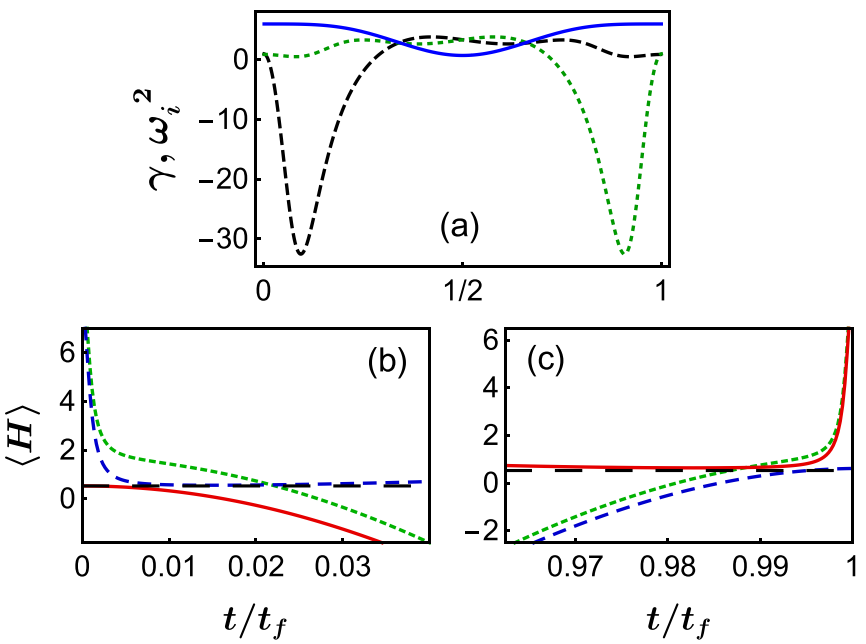

FIG. 3. (a) Control parameters, $\omega_{1}^{2}$ (dashed black), $\omega_{2}^{2}$ (shortdashed green) and $\gamma$ (solid blue) vs $t / t_{f}$, for an energy transfer from oscillator 1 to 2 . $\left\langle H_{1}\right\rangle$ in solid red, $\left\langle H_{2}\right\rangle$ in dashed blue, $\langle H\rangle$ in shortdashed green, and $\langle I\rangle$ in long-dashed black, for initial (b) and final (c) parts of the process. $\omega_{1}(0)^{2}=\omega_{2}\left(t_{f}\right)^{2}=1, \omega_{2}(0)^{2}=\omega_{1}\left(t_{f}\right)^{2}=$ 0.9 and $\gamma(0)=\gamma\left(t_{f}\right)=6 ; t_{f}=4$; the system starts in a (tensor) product state between the ground states of the uncoupled oscillators $H_{1}$ and $H_{2}$, not an eigenstate of the total Hamiltonian (1).

Coefficients $\{b, c\}_{1-5}$ are fixed so that the real reference trajectories satisfy the boundary conditions for $u_{1,2}\left(t_{b}\right)$ and its derivatives, and so that the frequencies $\omega_{i}(t)$ have the desired boundary values, which amounts to satisfying

$$
\begin{aligned}
& \ddot{u}_{1}^{I}(0)=-\omega_{1}(0)^{2}, \quad \ddot{u}_{1}^{I}\left(t_{f}\right)=\gamma\left(t_{f}\right) \sqrt{\frac{\omega_{1}(0)}{\omega_{2}\left(t_{f}\right)}}, \\
& \ddot{u}_{2}^{I}(0)=\gamma(0), \quad \ddot{u}_{2}^{I}\left(t_{f}\right)=\omega_{2}(t f) \sqrt{\omega_{1}(0) \omega_{2}\left(t_{f}\right)} .
\end{aligned}
$$

Note, from the expression of the frequencies

$$
\omega_{1,2}(t)^{2}=\frac{\gamma(t) u_{2,1}^{I}(t)-\ddot{u}_{1,2}^{I}(t)}{u_{1,2}^{I}(t)},
$$

that, even if the conditions in Eq. (20) are fulfilled, we may encounter indeterminacies at boundary times (some $u_{1,2}\left(t_{b}\right)$ become 0 ). Thus, we have to impose additional boundary conditions for consistency using L'Hopital's rule,

$$
\begin{aligned}
& u_{1}^{I(3)}\left(t_{f}\right)=0, \\
& u_{1}^{I(4)}\left(t_{f}\right)=-\gamma\left(t_{f}\right) \sqrt{\frac{\omega_{1}(0)}{\omega_{2}\left(t_{f}\right)}}\left[\omega_{1}\left(t_{f}\right)^{2}+\omega_{2}\left(t_{f}\right)^{2}\right], \\
& u_{2}^{I(3)}(0)=0, \\
& u_{2}^{I(4)}(0)=-\gamma(0)\left[\omega_{1}(0)^{2}+\omega_{2}(0)^{2}\right] .
\end{aligned}
$$

Coefficients $\{b, c\}_{6}$ are left yet undetermined. In the next step, we numerically solve the real equations of motion with the already designed control parameters for the initial conditions and find, again, numerically, the value of the coefficients that have been left free to satisfy the final boundary conditions.
Figure 3(a) displays the resulting evolution of the control parameters for a specific example in which the frequencies $\omega_{i}$ swap their boundary values and $\gamma(0)=\gamma\left(t_{f}\right)$. Figure 3(b) shows the expectation values of the total and the uncoupled Hamiltonians near the time boundaries, together with the constant expectation value of the invariant. Indeed $\left\langle H_{2}\left(t_{f}\right)\right\rangle=$ $\left\langle H_{1}(0)\right\rangle$, thus proving that our protocol transfers the state from one uncoupled oscillator at the initial time to the other one at the final time. Simililarly to what happens with the transversal energy in the wave guide, the invariant does not impose the value of the final total energy, which does not necessarily coincide with the initial one.

\section{DISCUSSION}

In some multidimensional systems with time-dependent control there are no point transformations that lead to uncoupled normal modes. Our main point here is that in these "coupled systems", invariants of motion may still guide us to inversely design the time dependence of the controls for driving specific dynamics.

This inversion procedure extends the domain of invariantbased engineering, which had been applied so far to 1D or uncoupled systems [4]. An important difference with respect to uncoupled systems is the diminished role of commutativity of Hamiltonian and invariant at boundary times. Commutativity, because of degeneracy, does not guarantee one-to-one mapping of eigenstates of the total Hamiltonian from initial to final configurations. One should then focus on the invariant itself for applications, and, if required, rely on design freedom to keep other variables, e.g., the total energy, controlled. An alternative to be explored is to make use of a second invariant corresponding to a linearly independent set of classical solutions of Eq. (7), $\left\{u_{1}^{\prime}(t), u_{2}^{\prime}(t)\right\}$, linearly independent with respect to $\left\{u_{1}(t), u_{2}(t)\right\}$ [27]. Imposing boundary conditions to the second set we would aim to control the second invariant as well, but the inversion problem becomes more demanding, as the number of conditions double, while the number of (common) controls remains the same.

As for further open questions, invariant-based engineering is known to be related to other STA approaches such as counterdiabatic driving for single oscillators [39]. It would be of interest to connect the current work with counterdiabatic driving for coupled oscillators [40,41]. Finally, other boundary conditions on the $u_{j}$ would allow to control other processes, different from the ones examined here.

\section{ACKNOWLEDGMENTS}

This work was supported by the Basque Country Government (Grant No. IT986-16), and by PGC2018-101355B-I00 (MCIU/AEI/FEDER,UE). E.T. acknowledges support from PGC2018-094792-B-I00 (MCIU/AEI/FEDER,UE), CSIC Research Platform PTI-001, and CAM/FEDER No. S2018/TCS- 4342 (QUITEMAD-CM). 
[1] J. P. Dowling and G. J. Milburn, Quantum technology: the second quantum revolution, Philos. Trans. R. Soc. London Ser. A 361, 1655 (2003).

[2] D. Kielpinski, C. Monroe, and D. J. Wineland, Architecture for a large-scale ion-trap quantum computer, Nature (London) 417, 709 (2002).

[3] E. Torrontegui, S. Ibáñez, S. Martínez-Garaot, M. Modugno, A. del Campo, D. Guéry-Odelin, A. Ruschhaupt, X. Chen, and J. G. Muga, Shortcuts to Adiabaticity, volume 62 of Advances In Atomic, Molecular, and Optical Physics (Elsevier, Amsterdam, 2013), p. 117.

[4] D. Guéry-Odelin, A. Ruschhaupt, A. Kiely, E. Torrontegui, S. Martínez-Garaot, and J. G. Muga, Shortcuts to adiabaticity: Concepts, methods, and applications, Rev. Mod. Phys. 91, 045001 (2019).

[5] X. Chen, A. Ruschhaupt, S. Schmidt, A. del Campo, D. Guéry-Odelin, and J. G. Muga, Fast Optimal Frictionless Atom Cooling in Harmonic Traps: Shortcut to Adiabaticity, Phys. Rev. Lett. 104, 063002 (2010).

[6] M. Demirplak and S. A. Rice, Adiabatic population transfer with control fields, J. Phys. Chem. A 107, 9937 (2003).

[7] H. R. Lewis and P. G. L. Leach, A direct approach to finding exact invariants for one-dimensional timedependent classical Hamiltonians, J. Math. Phys. 23, 2371 (1982).

[8] A. R. Urzúa, I. Ramos-Prieto, M. Fernández-Guasti, and H. M. Moya-Cessa, Solution to the time-dependent coupled harmonic oscillators hamiltonian with arbitrary interactions, Quant. Rep. 1, 82 (2019).

[9] M. Palmero, R. Bowler, J. P. Gaebler, D. Leibfried, and J. G. Muga, Fast transport of mixed-species ion chains within a Paul trap, Phys. Rev. A 90, 053408 (2014).

[10] M. Palmero, S. Martínez-Garaot, J. Alonso, J. P. Home, and J. G. Muga, Fast expansions and compressions of trapped-ion chains, Phys. Rev. A 91, 053411 (2015).

[11] M. Palmero, S. Martínez-Garaot, U. G. Poschinger, A. Ruschhaupt, and J. G. Muga, Fast separation of two trapped ions, New J. Phys. 17, 093031 (2015).

[12] M. Palmero, S. Martínez-Garaot, D. Leibfried, D. J. Wineland, and J. G. Muga, Fast phase gates with trapped ions, Phys. Rev. A 95, 022328 (2017).

[13] T. Sägesser, R. Matt, R. Oswald, and J. P. Home, Robust dynamical exchange cooling with trapped ions, New J. Phys. 22, 073069 (2020).

[14] I. Lizuain, M. Palmero, and J. G. Muga, Dynamical normal modes for time-dependent Hamiltonians in two dimensions, Phys. Rev. A 95, 022130 (2017).

[15] I. Lizuain, A. Tobalina, A. Rodriguez-Prieto, and J. G. Muga, Fast state and trap rotation of a particle in an anisotropic potential, J. Phys. A 52, 465301 (2019).

[16] R. Barends, J. Kelly, A. Megrant, D. Sank, E. Jeffrey, Y. Chen, Y. Yin, B. Chiaro, J. Mutus, C. Neill, P. O’Malley, P. Roushan, J. Wenner, T. C. White, A. N. Cleland, and J. M. Martinis, Coherent Josephson Qubit Suitable for Scalable Quantum Integrated Circuits, Phys. Rev. Lett. 111, 080502 (2013).

[17] M. A. Rol, F. Battistel, F. K. Malinowski, C. C. Bultink, B. M. Tarasinski, R. Vollmer, N. Haider, N. Muthusubramanian, A. Bruno, B. M. Terhal, and L. DiCarlo, Fast, High-Fidelity
Conditional-Phase Gate Exploiting Leakage Interference in Weakly Anharmonic Superconducting Qubits, Phys. Rev. Lett. 123, 120502 (2019).

[18] B. Peropadre, D. Zueco, F. Wulschner, F. Deppe, A. Marx, R. Gross, and J. J. García-Ripoll, Tunable coupling engineering between superconducting resonators: From sidebands to effective gauge fields, Phys. Rev. B 87, 134504 (2013).

[19] J. J. García-Ripoll, A. Ruiz-Chamorro, and E. Torrontegui, Quantum Control of Frequency-Tunable Transmon Superconducting Qubits, Phys. Rev. Appl. 14, 044035 (2020).

[20] Y. Chen, C. Neill, P. Roushan, N. Leung, M. Fang, R. Barends, J. Kelly, B. Campbell, Z. Chen, B. Chiaro, A. Dunsworth, E. Jeffrey, A. Megrant, J. Y. Mutus, P. J. J. O’Malley, C. M. Quintana, D. Sank, A. Vainsencher, J. Wenner, T. C. White, M. R. Geller, A. N. Cleland, and J. M. Martinis, Qubit Architecture with High Coherence and Fast Tunable Coupling, Phys. Rev. Lett. 113, 220502 (2014).

[21] D. Kleckner, B. Pepper, E. Jeffrey, P. Sonin, S. M. Thon, and D. Bouwmeester, Optomechanical trampoline resonators, Opt. Express 19, 19708 (2011).

[22] K. Zhang, F. Bariani, and P. Meystre, Quantum Optomechanical Heat Engine, Phys. Rev. Lett. 112, 150602 (2014).

[23] M. Aspelmeyer, T. J. Kippenberg, and F. Marquardt, Cavity optomechanics, Rev. Mod. Phys. 86, 1391 (2014).

[24] H. Goldstein, C. Poole, and J. Safko, Classical Mechanics (Addison-Wesley, Reading, MA, 2002).

[25] M. Fernández-Guasti and A. Gil-Villegas, Orthogonal functions invariant for the time-dependent harmonic oscillator, Phys. Lett. A 292, 243 (2002).

[26] M. Fernández-Guasti and H. Moya-Cessa, Solution of the Schrödinger equation for time-dependent 1D harmonic oscillators using the orthogonal functions invariant, J. Phys. A 36, 2069 (2003).

[27] K.-E. Thylwe and H. J. Korsch, The 'Ermakov-Lewis' invariants for coupled linear oscillators, J. Phys. A 31, L279 (1998).

[28] O. Castanos, R. Lopez-Pena, and V. I. Man'ko, Noether's theorem and time-dependent quantum invariants, J. Phys. A 27, 1751 (1994).

[29] V. I. Arnold, Mathematical Methods of Classical Mechanics, 2nd ed. (Springer-Verlag, Berlin, 1989).

[30] C. Cohen-Tannoudji, B. Diu, and F. Laloe, Quantum Mechanics (Wiley, New York, 1991).

[31] J. I. Cirac, P. Zoller, H. J. Kimble, and H. Mabuchi, Quantum State Transfer and Entanglement Distribution among Distant Nodes in a Quantum Network, Phys. Rev. Lett. 78, 3221 (1997)

[32] M. Christandl, N. Datta, A. Ekert, and A. J. Landahl, Perfect State Transfer in Quantum Spin Networks, Phys. Rev. Lett. 92, 187902 (2004).

[33] D. N. Matsukevich and A. Kuzmich, Quantum state transfer between matter and light, Science 306, 663 (2004).

[34] P. Kurpiers, P. Magnard, T. Walter, B. Royer, M. Pechal, J. Heinsoo, Y. Salathé, A. Akin, S. Storz, J. Besse, S. Gasparinetti, A. Blais, and A. Wallraff, Deterministic quantum state transfer and remote entanglement using microwave photons, Nature (London) 558, 264 (2018). 
[35] A. S. M. de Castro and V. V. Dodonov, Squeezing exchange and entanglement between resonantly coupled modes, J. Russ. Laser Res. 23, 93 (2002).

[36] A. S. M. de Castro, V. V. Dodonov, and S. S. Mizrahi, Quantum state exchange between coupled modes, J. Opt. B 4, S191 (2002).

[37] A. S. M. de Castro and V. V. Dodonov, Purity and squeezing exchange between coupled bosonic modes, Phys. Rev. A 73, 065801 (2006).

[38] S. González-Resines, D. Guéry-Odelin, A. Tobalina, I. Lizuain, E. Torrontegui, and J. G. Muga, Invariant-Based Inverse
Engineering of Crane Control Parameters, Phys. Rev. Appl. 8, 054008 (2017).

[39] X. Chen, E. Torrontegui, and J. G. Muga, Lewis-Riesenfeld invariants and transitionless quantum driving, Phys. Rev. A 83, 062116 (2011).

[40] C. W. Duncan and A. del Campo, Shortcuts to adiabaticity assisted by counterdiabatic Born-Oppenheimer dynamics, New J. Phys. 20, 085003 (2018).

[41] T. Villazon, A. Polkovnikov, and A. Chandran, Swift heat transfer by fast-forward driving in open quantum systems, Phys. Rev. A 100, 012126 (2019). 